\title{
Editor's note on Volume 5 Issue 3
}

\section{Pollen K F Yeung}

Professor of Pharmacy and Medicine Dalhousie University, Canada, Tel: 902-4943845; E-mail: Pollen.Yeung@Dal.Ca

\section{Background}

Cardiovascular Pharmacology Open Access which focuses on diagnosis, characterization, and pharmacological management of cardiovascular diseases including with complementary medicine. It mainly aims to promote international dialogue and collaboration on health issues between practicing clinicians and basic research scientists in the area of cardiovascular pharmacology.

The current issue of the journal "Cardiovascular Pharmacology Open Access" majorly highlights the management of cardiovascular diseases. The objective is to improve clinical practice and to expand and deepen the understanding of health and health care related to management of cardiovascular diseases. Its broad focus makes it an excellent resource for researchers to disseminate their latest research findings.

\section{In this Issue}

Primarily in the Editorial, I discussed the importance of gender effect in the management of Cardiovascular Disease.

Vikrant Vijan et al., published their research articles related to retrospective analysis comparing two antiplatelet agents (ticagrelor vs clopidogrel) in patients having acute coronary syndromes and the other is an Prospective study of Dyspnea in post Percutaneous Transluminal Coronary Angioplasty (PTCA) patients.

John F Beltrame et al., published their article on Endothelial denudation of isolated human internal mammary artery segments.
They conducted controlled comparative study assessing both structural and functional endpoints of endothelial denudation techniques.

Carmine Marini et al., conducted Meta-Analysis of Randomized Controlled Trials for evaluating possible benefits of endovascular therapy.

Yunde Liu et al., written a review on early subclinical biomarkers in onco-cardiology to prevent cardiac death. They summarized Blood biomarkers provide non-invasive, practical and reliable methods to identify high risk of cancer patients in cardiac functions, especially asymptomatic events.

Giuseppe Di Stolfo et al., submitted a Case Report on Over the counter drugs and long QT syndrome. They clearly explained the advantage and risk of self medication by over the counter (OTC) drugs, in relationship to QTc prolongation, unrevealing concealed QT syndrome and Sudden Arrhythmia Deaths Syndrome (SADS).

Finally Idrus Alwi et al., explained the Effects of Curcumin Against the Inflammatory Response in Patients with Acute Coronary Syndrome. They report on the anti-inflammatory effects of curcumin for patients with ACS by measuring their hsCRP (highly sensitive C-Reactive Protein) levels. This is significant because when Acute Myocardial Infarct (AMI) or ACS occurs, the patient experiences an increase in the level of hsCRP, and it also may affect the onset pattern of AMI.

Thus this current issue provides interest to readers in the areas of General Cardiology, Interventional Cardiology, and nutrition. 